\title{
CONCENTRATION OF MAGNETIC FIELDS IN THE DEEP CONVECTION ZONE
}

\author{
G. W. SimON \\ (Sacramento Peak Observatory, \\ Sunspot, N.M., U.S.A.)
}

\author{
and
}

N.O. WeISS

(Dept. of Applied Mathematics

and Theoretical Physics,

Silver Street, Cambridge, England)

\begin{abstract}
A BSTRACT
The strong magnetic fields observed between supergranules indicate that there must be subphotospheric convection in cells with a preferred diameter of about $30000 \mathrm{~km}$. Orthodox mixing length theory assumes that the dimensions of cells are limited by the density scale-height. This is adequate for explaining granules but cannot account for supergranulation. A model is therefore proposed in which cellular motions extend over several scale-heights. In addition to granules and supergranules. this model predicts a third characteristic scale of motion, with giant cells around $300000 \mathrm{~km}$ in diameter. These cells may produce a pattern of magnetic fields like that suggested by Bumba and Howard for complexes of activity.
\end{abstract}

\section{Introduction}

We shall first discuss the structure of convection in the Sun's hydrogen convection zone and, in particular, the scale of the motions occurring in it. Then we shall briefly attempt to relate this structure to magnetic-field patterns observed at the surface of the Sun.

Granules and supergranules show cellular motion on two scales in the solar atmosphere. The average diameter of a granule is around $2000 \mathrm{~km}$, while the average diameter of supergranules is $30000 \mathrm{~km}$. Moreover, the supergranulation is apparently caused by subphotospheric convection. The photospheric magnetic fields appear to have been swept aside and concentrated at the boundaries of supergranules; but the kinetic energy of motion in supergranules corresponds to a field of about 20 gauss, whereas Sheeley (1966) has measured strengths of several hundred gauss. Again, the tendency of sunspots to form at junctions of supergranules (Bumba and Howard, 1965a), coupled with the size of sunspot fields and the scale of sunspot groups, indicates that supergranular motions must represent deep-seated phenomena. If, then, we have a satisfactory model of correction in the Sun, it must explain not only granules but also the supergranulation.

\section{Convection Cells extending over Several Scale Heights}

Let us consider first the orthodox theory of stellar convection. If we had only a

Kiepenheuer (ed.), Structure and Development of Solar Active Regions, 108-111. C I.A.U. 
layer of incompressible fluid and could ignore the boundaries, we could describe convection in terms either of interacting bubbles or of eddies: in each case, the scale of motion would be comparable with the depth of the convective layer. However, in the Sun the gas is compressible and the density varies by a factor of order $10^{5}$ over the convective zone. Because of continuity the motion would become very complicated: a small motion at the bottom of the layer would require very rapid motion at the top. So Biermann and Schwarzschild suggested that the local density scale-height should be taken as the mixing length or, effectively, the characteristic scale for convective eddies. Thus we obtain a pattern of motion whose scale is determined by the local scale-height. When we study a model convective zone obtained in this way, we find that all its properties vary smoothly with depth. It is impossible to produce any single distance typical of the convective zone, let alone explain the predominance of eddies with a scale of $30000 \mathrm{~km}$. The orthodox model accounts nicely for granulation but it cannot explain the existence of supergranules.

To cope with this difficulty we were therefore led to postulate that $30000 \mathrm{~km}$ is indeed in some sense a typical distance. We suppose that convection occurs in cells with a diameter of $30000 \mathrm{~km}$ over at any rate a substantial part of the convection zone.

We have discussed two crude physical models of convection in a compressible atmosphere, in order to have some quantitative results (Simon and Weiss, 1968). First of all, we considered convection cells extending over many scale-heights in an atmosphere whose scale-height was independent of depth. Owing to the stratification, the motion is dominated by rising blobs of gas. Very little of the fluid originating at the bottom of the cell will reach the top but since the fluid rises adiabatically it will carry a greater temperature excess than would be possible for blobs originating locally. The mass flux at the top of the cell is relatively small and the argument that continuity demands rapid motions does not apply. The superadiabatic gradient, $\beta_{1}$, required to transport a fixed convective flux can be crudely estimated by balancing non-linear dissipation against work done by the buoyancy forces. This can be compared with the superadiabatic gradient, $\beta_{0}$, obtained from the orthodox model. We found that

$$
\beta_{1} \approx \frac{1}{3} \beta_{0}
$$

and cells extending over many scale-heights are therefore more efficient at transporting heat.

In the solar atmosphere the scale-height is not constant. Instead, it increases with depth, $z$, below the surface. As a result, horizontal velocities dominate the motion in the upper regions of a cell extending over many scale-heights. We considered a polytropic atmosphere with a relationship

$$
P=K \rho^{\Gamma}
$$

between the pressure, $P$, and the density, $\rho$, and a polytropic index

$$
m=(\Gamma-1)^{-1} \text {. }
$$


Much of the solar convective zone is fairly well described by a polytrope with $m=\frac{3}{2}$. In such an atmosphere we would expect cells of diameter $2 L$ to be generated where the local scale-height was comparable with $L$. But the level to which motions on this scale can penetrate is now limited. Using the same physical arguments, we would expect cells of diameter $2 L$ to extend from $z \approx \frac{1}{10} L$ to $z \approx L$. Thus they would cover 3 to 4 scale-heights or a range of about 30 in the density $\rho$.

This crude physical treatment indicates that we might expect to find cellular motions extending over, say, four density scale-heights rather than just one as in the orthodox model. This result should be supported by detailed numerical experiments but we may still ask the question: What can we infer about the pattern of convection in the Sun?

\section{Convection in the Sun}

The solar convection zone is currently estimated to be about $150000 \mathrm{~km}$ in depth; it is unlikely that its overall structure would be significantly affected by changes in the convection model used. The detailed motion will be dominated by rising blobs which spread out and interact to produce eddy-like patterns. If these typically cover four scale-heights, we might expect eddies generated at the base of the convective zone to penetrate to a depth of about $20000 \mathrm{~km}$ and to have a characteristic diameter of around $300000 \mathrm{~km}$; motions originating at $z=20000 \mathrm{~km}$ might have a diameter of $30000 \mathrm{~km}$ and penetrate to $z \approx 3000 \mathrm{~km}$; above that level radiative transfer becomes important, so all scales from $4000 \mathrm{~km}$ downwards might be visible at the surface.

We do not, of course, suggest that the convective zone is separated into three discrete regions. However, we predict that the distribution of cell sizes should show three distinct peaks: one at about $2000 \mathrm{~km}$, corresponding to the granulation; another at $30000 \mathrm{~km}$, corresponding to supergranules; and a third around $300000 \mathrm{~km}$, corresponding to giant cells in the deep convective zone. That is to say, a convection model that explains both granules and the preferred dimensions of supergranules will also predict a third preferred scale, comparable with the depth of the convective zone itself.

For both supergranules and giant cells we can estimate typical values of the vertical component of the velocity, $w$, the horizontal component, $u$, and the timescale $\tau=L / w$. These values are shown in Table 1 . In fact, the values of $w$ are effectively the same as those found using orthodox mixing-length theory near the lower boundaries of the eddies.

\section{Table 1}

\section{Cellular motions in the convective zone}

$\begin{array}{ccccc}L & \stackrel{w}{L} & u & \boldsymbol{\tau} \\ (\mathrm{km}) & (\mathrm{km} / \mathrm{sec}) & (\mathrm{km} / \mathrm{sec}) & \text { (days) } & \text { (gauss) }\end{array}$

Supergranules

$1.5 \times 10^{4}$

$$
0 \cdot 3
$$

$1.5 \times 10^{5}$

$0 \cdot 1$

$0 \cdot 6$

$0 \cdot 2$ 


\section{Interaction of Convection and Magnetic Fields}

Over most of a cell the horizontal velocity acts in the same sense, outwards from the centre; in the upper part it will be greater than the vertical component. This pattern of motion will therefore be particularly effective in sweeping aside magnetic flux and concentrating it at the edges of the cell. This process will be halted when the energy of the concentrated field is comparable with the kinetic energy of the horizontal motion. This equipartition field varies slowly with depth for a given value of $L$. Typical values are given in Table 1.

The formation and development of individual active centres is indeed closely related to the supergranulation pattern. (Bumba and Howard, 1965a). If giant cells are present, they should also have observable effects on magnetic fields at the surface of the Sun. Some structure with a characteristic scale of about $300000 \mathrm{~km}$ should be present. As Professor Kiepenheuer has already pointed out, Bumba and Howard have indeed suggested that complexes of activity (Bumba and Howard, 1965b) do show such a structure and that it may persist over many solar rotations. If this pattern is present, the growth and migration of such complexes may be related to cellular motions at the base of the convection zone. Complexes of activity should form at junctions of giant cells, while individual active centres are controlled by supergranular motions. The complexes might be swept aside as the pattern of motions varies near the bottom of the convection zone. Close study of their development may thus enable us to investigate convection $100000 \mathrm{~km}$ below the surface of the Sun.

\section{References}

Bumba, V., Howard, R. F. (1965a) Astrophys. J., 141, 1492.

Bumba, V., Howard, R. F. (1965b) Astrophys. J., 141, 1502.

Sheeley, N. R., Jr. (1966) Astrophys. J., 144, 723.

Simon, G.W., Weiss, N.O. (1968) to be published.

\section{DISCUSSION}

Kiepenheuer: How does the lifetime of your model of a convective cell agree with the observed lifetime of a supergranulum (about one day)?

Weiss: The turnover time for our supergranular cells is about 10 hours; for the giant cells it is around 10 days. So a giant cell might have a lifetime of about 20 days, corresponding to the observed lifetime of supergranules, which is twice this turnover time.

Acton: How can a toroidal magnetic field as postulated in the Babcock theory be compatible with the radial convection field?

Weiss: The convection is a three-dimensional process and the horizontal components of the velocity may be greater than the vertical components. However, some concentration of the toroidal field below the bottom of the convection zone may occur, as was suggested by Spitzer.

Beckers: Have you estimated the temperature fluctuations in the photosphere resulting from your convection cells?

Weiss: Not precisely. One would expect fluctuations of, say, $200^{\circ}$ at the top of supergranular cells, but the granulation would make any variation at the photospheric level much smaller. 